\title{
Der Leistungsauftrag als Grundlage für die Gestaltung der Notfallmedizin
}

\section{Urs Genewein ${ }^{a, b}$, \\ Marcel Jakob ${ }^{b, c}$, \\ Roland Bingisserc, \\ Stephan Burla , \\ Michael Heberer ${ }^{a, d}$}

a Forschungsgruppe Management, Institut für Chirurgische Forschung und Spitalmanagement, Universitätsspital Basel

b Behandlungszentrum Bewegungsapparat, Universitätsspital Basel

c Notfallstation, Universitätsspital Basel

d Institut für Chirurgische Forschung und Spitalmanagement, Universitätsspital Basel
Korrespondenz:

Prof. Dr. med. Michael Heberer, FACS, MBA

Institut für Chirurgische Forschung und Spitalmanagement

Mitglied der Spitalleitung

Universitätsspital Basel

Hebelstrasse 20

CH-4031 Basel

mheberer@uhbs.ch

\section{Zusammenfassung}

Notfallmedizin unterscheidet sich hinsichtlich Dringlichkeit, Leistungsbreite und Komplexität (Variabilität der Nachfrage, medizinische Spezialisierung, Mensch-Maschinen-Schnittstelle, Entscheidungen unter Unsicherheit) von anderen medizinischen Aufgabengebieten. Sie erfordert deshalb einen spezifischen Leistungsauftrag. - Wir haben notfallmedizinische Leistungsaufträge anhand der Literatur und einer Befragung der grossen schweizerischen Spitäler analysiert. - Die aktuellen Leistungsaufträge bieten den Notfallstationen eine wenig konkrete Handlungs- und Entscheidungsgrundlage; allerdings stellt die Literatur Kennzahlen zur Verfügung, um einen quantitativen Auftrag hinsichtlich Leistungsbreite (fachliches Spektrum), Leistungstiefe (Prozessgestaltung), Leistungsqualitäten (Wartezeiten, Zufriedenheitsziele) und Leistungsvolumina (Ressourcenbedarf und -auslastung) zu definieren. Es ist zu fordern, die Leistungsfähigkeit der Notfallmedizin durch derart spezifische Leistungsaufträge $\mathrm{zu}$ unterstützen.

\section{Einleitung}

Notfallstationen und klinische Notfallmedizin sind in der Kritik. Überlastung [1, 2], verzögerte Übernahmen von Notfallpatienten in die stationäre und poststationäre Betreuung [2, 3], Qualitäts- [4] und Servicemängel (Wartezeiten) [5] sowie überlastetes Personal [6] sind Symptome einer Krise der klinischen Notfallmedizin, deren Ursachen bekannt sind:

1. Die Nachfrage nach notfallmedizinischen Leistungen der Spitäler nimmt in den industrialisierten Ländern zu. Für diese Entwicklung spielt in der Schweiz die Leistungsverschiebung vom Hausarzt zur Notfallstation des Spitals eine wesentliche Rolle [7], während in den USA die Versicherungssituation der Bevölkerung ursächlich ist [8].

2. Die Spezialisierung steigert einerseits die Leistungsfähigkeit der Notfallmedizin; andererseits kann kaum ein Spital noch sämtliche Spezialisten während 24 Stunden vor Ort bereithalten: Es resultiert eine Diskrepanz zwischen der patientenseitigen Erwartung der sofortigen Behandlung durch den Spezialisten und dem Angebot des Spitals.

3. Die Variabilität der Nachfrage nach Notfallleistungen ist bereits unter Normalbedingungen erheblich [9]. In besonderen Situationen (Grippe, Glatteis) und bei Katastrophen (Massenunfälle, Terrorismus) müssen Not-

\section{Défi des services d'urgence: le mandat de prestations comme base pour la formation de l'aide médicale urgente}

L'aide médicale urgente (AMU) se différencie des autres disciplines médicales en raison de l'aspect d'urgence et de l'éventail de ses prestations ainsi que de par sa complexité (variabilité de la demande, spécialisation médicale, interface hommemachine, décisions prises malgré une certaine incertitude). Elle requiert par conséquent un mandat de prestations spécifique. - Nous avons analysé des mandats de prestations d'AMU sur la base de la littérature et d'une enquête auprès des grands hôpitaux suisses. - Les mandats de prestations ne constituent pas une base d'action ou de décision suffisamment concrète pour les services d'urgence. La littérature fournit toutefois des indicateurs permettant de définir un mandat quantitatif pour ce qui est de l'éventail des prestations (éventail technique), de leur envergure (organisation des processus), de leurs qualités (temps d'attente, objectifs de satisfaction) et de leurs volumes (besoin et occupation des ressources). Il est indispensable que de tels mandats de prestations bien spécifiques puissent contribuer à la performance de l'aide médicale d'urgence. 
fallstationen zusätzliche Kapazitäten rasch aufbauen können [10].

4. Wirtschaftlicher Druck und Arbeitszeitverkürzungen sind weitere Bedingungen, mit denen die Notfallmedizin zurechtkommen muss [1].

Diesen Herausforderungen der Notfallmedizin kann die Selbststeuerung durch die Normen des ärztlichen und pflegerischen Handelns nicht gewachsen sein [11-13]. Konkrete Leistungsaufträge als Grundlage von Organisation und Bereitstellung der erforderlichen Ressourcen sind unverzichtbar. Wir haben deshalb die Leistungsaufträge von Notfallstationen untersucht.

\section{Methoden \\ Literaturanalyse}

Die Datenbank PubMed wurde für den Zeitraum von Januar 2000 bis Februar 2007 nach Literaturstellen durchsucht, in denen der Suchbegriff «Emergency Medical Services» («Major Topic» [MAJR]) durch folgende Unterschlagwörter (Subheadings) qualifiziert wurde: classification, manpower, organization \& administration, standards, statistics \& numerical data, supply \& distribution, trends oder utilization. Die resultierenden Arbeiten wurden auf die Fragen des Leistungsauftrags und der Indikatoren der Auftragserfüllung untersucht [14].

\section{Feldstudie}

Mit Genehmigung der zuständigen Gesundheitsdirektionen wurden im Februar 2007 die Leistungsaufträge von 19 grossen schweizerischen Spitälern anhand eines Fragebogens analysiert. Eingeschlossen wurden die 5 Universitätsspitäler und die 14 grossen Spitäler, die von der FMH für die Fächer Chirurgie und Innere Medizin als Weiterbildungsstätten der Kategorie A geführt werden. Anonymität wurde zugesichert.

\section{Ergebnisse \\ Literaturanalyse zum Leistungsauftrag}

Die Leistungsaufträge von Notfallstationen umfassen die klinische Versorgung, die Vorsorge für besondere Ereignisse (Katastrophen) sowie Lehre und Forschung (Tab. 1). Der klinische Versorgungsauftrag schliesst Aufnahme, Diagnostik, Behandlung und Verlegung zur weiteren Behandlung ein. Diese Schritte sollen aus medizinischen, ökonomischen und servicebezogenen Gründen in möglichst kurzer Zeit erledigt werden [15-18].

Die veröffentlichten Leistungsaufträge beinhalten kaum quantitative Zielvorgaben. Eine Ausnahme stellt der Plan 2000 des britischen National Health Service dar, mit dem für das Jahr 2004 die maximale Wartezeit in Notfallstationen mit 4 Stunden und die Anzahl der Patienten, die innert 20 Minuten nach Aufnahme eine notwendige Thrombolyse erhalten, mit $75 \%$ spezifiziert werden [17].

Dass solche konkreten Ziele Wirkung entfalten, wird beispielsweise durch Vereinbarungen zwischen Notfallstationen und weiterbehandelnden Abteilungen in Kanada belegt: Diese Vereinbarung führte zu einer Reduktion der durchschnittlichen Aufenthaltsdauer in der Notfallstation von 13,8 auf 5,9 Stunden [15].

Einige Leistungsaufträge von Notfallstationen geben Ziele für Forschung und Weiterbildung vor [19, 20], andere definieren auch Anforderungen an die Leistungsbereitschaft unter besonderen Bedingungen (Katastrophen) [21-23].

Dazu stellt die Literatur ein breites Spektrum von Leistungsindikatoren zur Verfügung (Tab. 2). Technische Kennzahlen werden in den Kategorien «zeitliche Definitionen, Zeitintervalle, Prozessdefinitionen, Raumdefinitionen» [24] oder «input, throughput and output» [18] strukturiert. Auch Kennzahlen zu Patienten-

Tabelle 1

Leistungsauftrag von Notfallstationen (Literaturrecherche 2000-2007).

\section{Kriterium}

Klinische Notfallversorgung

Bereitschaft

(= klinische Vorhalteleistung)

Lehre und Forschung

\section{Leistungsauftrag}

Notfallaufnahme, Triage und Behandlung ohne definierten Endpunkt Ankunft bis Entlassung von der Notfallstation (LOS als Kriterium) Notfallaufnahme, Triage, NF-Diagnostik und Behandlung, Verlegung Notfallwartezeit im Mittel 75 min und maximal 4 h (Ziel 2004) Anpassung der Aufnahmekapazität

Qualitätsverbesserung durch Kooperation mit Wissenschaftlern Teamtrainingsprogramme für Notfallmedizin
Quelle

12 Kantonsspitäler, Schweiz [28] 273 Emergency Departments, USA [16] USA [18] NHS-Programm, UK [17] Emergency Departments, USA [21-23]

Pennsylvania, USA, Health Network [19] Providence, USA [20] 
sicherheit und medizinischen Ergebnissen werden berücksichtigt (good quality of care) [25].

\section{Leistungsaufträge}

\section{schweizerischer Notfallstationen}

«Das Spital X sichert mit einer interdisziplinären Notfallstation rund um die Uhr die Versorgung spitalinterner und spitalexterner Notfälle für alle Fachbereiche. Dabei handelt es sich um die Aufnahme, Abklärung und Erstbehandlung von Schwerverletzten, Verunfallten und Kranken, die einer notfallmässigen Abklärung und Therapie bedürfen. Zudem dient die Notfallstation der Primärversorgung von kleinen Verletzungen.»
Dies ist ein typischer Leistungsauftrag einer schweizerischen Notfallstation.

14 von 19 Spitälern beantworteten die Umfrage. 3 der 14 Spitäler gaben an, über keinen expliziten Leistungsauftrag für die Notfallstationen zu verfügen. Die Leistungsaufträge der verbleibenden 11 Spitäler wurden analysiert (Tab. 3).

8 von 11 Leistungsaufträgen wurden durch den Kanton definiert. In 2 Fällen wurde dieser Auftrag durch die Spitalleitung detailliert. 3 Leistungsaufträge wurden ausschliesslich durch die Spitalleitungen definiert. Alle Leistungsaufträge wurden während der zurückliegenden 10 Jahre

Tabelle 2

Leistungsindikatoren von Notfallstationen.

\begin{tabular}{|c|c|c|}
\hline Kategorie & Indikator & Quelle \\
\hline Leistung (Throughput, Umsatz) & Insgesamt behandelte Patienten und erbrachte Leistungen & [24] \\
\hline \multirow[t]{4}{*}{ Prozess } & Wartezeiten & [28] \\
\hline & $\begin{array}{l}\text { Behandlungsdauer in der Notfallstation, sogenannte «turnaround time» } \\
\text { (Aufnahme bis Entlassung oder Verlegung; LOS) }\end{array}$ & {$[16,22,29]$} \\
\hline & Aufnahmekapazität (stündlich, täglich, innert definierter Zeit) & {$[21,22,27]$} \\
\hline & Anzahl verfügbarer Betten & {$[30]$} \\
\hline \multirow[t]{3}{*}{ Medizinisches Ergebnis } & Triagequalität (Richtigkeit medizinischer Entscheidungen) & {$[28,29]$} \\
\hline & Überlebensraten, Sterblichkeit (Todesfälle/1000 Zuweisungen) & {$[30]$} \\
\hline & Wiedereintritte innert 7 Tagen & [15] \\
\hline \multirow[t]{2}{*}{ Kundenzufriedenheit } & Patientenzufriedenheit (Erfahrung versus Erwartung) & {$[28,31]$} \\
\hline & Patienten, die NFS ohne Untersuchung und Behandlung verlassen haben & [24] \\
\hline \multirow[t]{3}{*}{ Bereitschaft und Vorhaltung } & Aufnahmekapazität (statisch und dynamisch) & {$[21,32]$} \\
\hline & Katastrophenmanagement (best practice guidelines: Protokolle definiert) & [33] \\
\hline & Katastrophenübungen durchgeführt & [33] \\
\hline \multirow[t]{2}{*}{ Wirtschaftliches Ergebnis, Produktivität } & Kosten pro Patient & [30] \\
\hline & Patienten pro FTE & [30] \\
\hline
\end{tabular}

\section{Tabelle 3}

Leistungsaufträge schweizerischer Notfallstationen. Die Anzahl der Antworten bezieht sich auf 19 angefragte Spitäler. Von 14 Spitälern wurde die Anfrage beantwortet; von diesen verfügten 11 Spitäler über einen Leistungsauftrag, der analysiert werden konnte.

\begin{tabular}{|c|c|c|c|}
\hline \multirow[t]{2}{*}{ Qualitätsdimension } & \multirow[t]{2}{*}{ Kriterium } & \multicolumn{2}{|c|}{ Ausprägung (= Erwähnungen in den Leistungsaufträgen) } \\
\hline & & Keine Erwähnung & Qualitative Beschreibung \\
\hline \multirow[t]{3}{*}{ Klinische Leistung } & Leistungsspektrum (Anzahl Fachgebiete) & 1 & 10 \\
\hline & Fallzahlen & 7 & 4 \\
\hline & Patienteninformation & 4 & 7 \\
\hline \multirow[t]{3}{*}{ Bereitschaft (Vorhalteleistung) } & Aufnahmebereitschaft & 3 & 8 \\
\hline & Versorgungssicherheit & 5 & 6 \\
\hline & Katastrophenplan & 7 & 4 \\
\hline Prozess & Zeitliche Vorgaben & 11 & 0 \\
\hline Medizinisches Ergebnis & & 11 & 0 \\
\hline Patientenzufriedenheit & & 11 & 4 \\
\hline Lehre & & 6 & 5 \\
\hline Forschung & & 7 & 4 \\
\hline Wirtschaftliches Ergebnis & Leistungserfassung & 8 & 3 \\
\hline
\end{tabular}


Abbildung 1

Integrativer Auftrag der Notfallstation: Notfallbehandlung und Vorbereitung zur stationären oder ambulanten Weiterbehandlung durch Spezialisten und Generalisten. Die Notfallstation wird zur Drehscheibe mit definiertem Leistungsauftrag (Service level agreements) und Entscheidungsstelle für die zeitlich nachgeordneten Spezialisten.

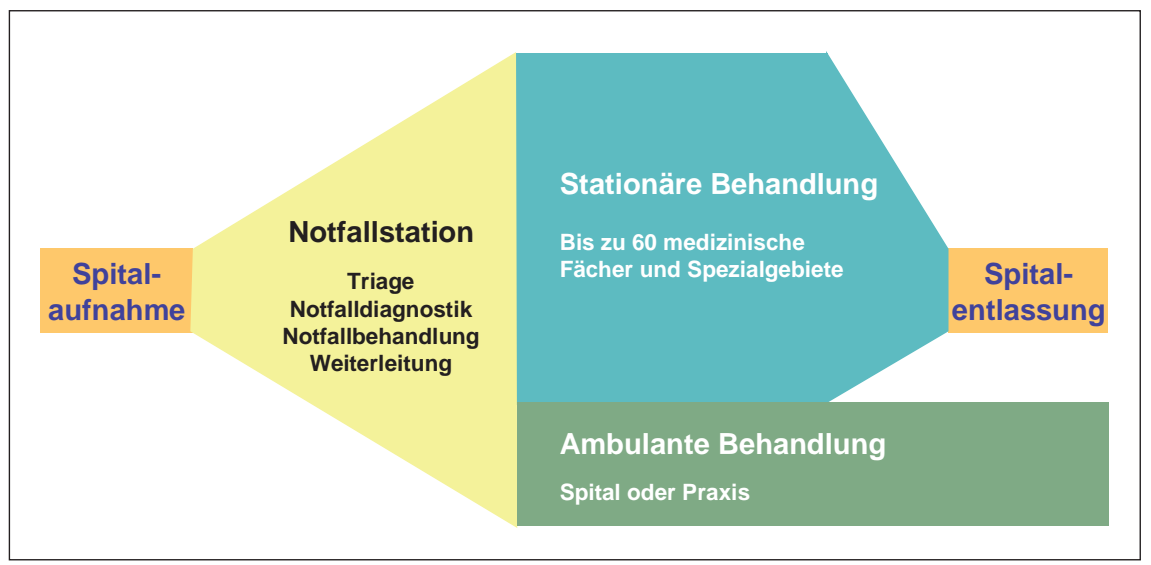

erstellt, 4 Leistungsaufträge waren weniger als 2 Jahre alt.

In allen Leistungsaufträgen dominierten qualitative Ziele. Diese konnten den Dimensionen klinische Leistung, Bereitschaftsleistung, Lehre und Forschung sowie wirtschaftliches Ergebnis zugeordnet werden. Insbesondere wurden qualitative Leistungsziele zu Patientenversorgung (regelhafte Basisversorgung, Versorgung von Schwerverletzten, Fasttrack für Leichtverletzte in 9 Aufträgen) und Katastrophenbereitschaft (6 Aufträge) formuliert. In 9 Leistungsaufträgen wurde die Struktur der Notfallstation als unidisziplinär, multidisziplinär oder multiprofessionell beschrieben. In 5 Leistungsaufträgen wurde die Führungsstruktur als multidisziplinäre oder getrennt ärztliche und pflegerische Führung festgelegt. Die Dimensionen Prozessqualität, medizinisches Ergebnis und Patientenzufriedenheit fanden Berücksichtigung in den Leistungsaufträgen.

Strukturelle Anforderungen wurden für die Verfügbarkeit der Notaufnahme (11 Aufträge), die Operationsbereitschaft (4 Aufträge) und die Infrastruktur zur Triage und Versorgung von Schwerverletzten (2 Aufträge) formuliert. Bereitschaftskapazitäten von Spezialisten wurden in einem Leistungsauftrag konkretisiert.

Quantitative Ziele, deren Erreichung über ein Kennzahlensystem nachvollziehbar wäre, wurden in den Leistungsaufträgen nicht festgelegt.

\section{Diskussion}

\section{Herausforderung Notfallstation}

In der Schweiz stellen Notfallstationen eine Anlaufstelle für alle unerwartet aufgetretenen medizinischen Beschwerden von Patientinnen und Patienten dar, für die diese sich an die Notfallstation (und nicht an den Hausarzt oder eine andere medizinische Einrichtung) wenden. Zusätzlich existieren spezialisierte Notfallstationen, deren Leistungen sich auf umschriebene Hilfeleistungen beispielsweise für Kinder oder in der Geburtshilfe beschränken.

Damit ist das Spektrum der Erkrankungen und Verletzungen, für die eine Notfallstation vorbereitet sein muss, breit. Zugleich ist die Nachfrage nach medizinischer Notfallbehandlung variabel und schwer zu prognostizieren. Weitere Merkmale, die die Komplexität der Notfallmedizin beschreiben, sind Technisierung (Mensch-Maschine-Schnittstelle) und wirtschaftlicher Druck (Effizienzziel). Die Notfallorganisation muss diese Komplexität beherrschen und dabei zwei zentrale Zielkonflikte lösen:

1. Einerseits ist die medizinische Spezialisierung die Basis der hohen Leistungsfähigkeit der Notfallmedizin. Andererseits kann das Potential der medizinischen Spezialisierung im Notfall nicht immer unmittelbar zur Verfügung gestellt werden, weil nicht alle Spezialisten während 24 Stunden an 365 Tagen vor Ort sein können.

2. Einerseits wird von Notfallstationen heute eine hocheffiziente und kostengünstige Dienstleistung erwartet. Andererseits sollen Notfallstationen auch Nachfragespitzen und Katastrophen bewältigen können.

\section{Leistungsauftrag und Leistungskennzahlen der Notfallstation}

Aus den genannten Ansprüchen lässt sich ein breiter Leistungsauftrag ableiten (Abb. 1): Dieser umfasst Aufnahme, Notfalldiagnostik und Initialbehandlung aller Notfallpatientinnen und -patienten. Dieser Auftrag wird oft mit einigen fachlichen Einschränkungen (beispielsweise inklusive oder exklusive Kinder oder gynäkologisch-geburtshilflicher Notfälle) im politischen Leistungsauftrag wiedergegeben.

Dieser qualitative Leistungsauftrag lässt viele Fragen offen. Undefiniert bleiben die Anforderungen an die medizinischen Qualitäten der Notfallbehandlung (Wartezeiten, Fehlerraten, Spezialistenpräsenz usw.), an den Abschluss der Notfallbehandlung (Entlassung oder Übergabe an den weiterbehandelnden Spezialisten), an die prognostischen Fähigkeiten (Nachfragevorhersage in Abhängigkeit von Wetter und Grippewelle) und an die Personalrekrutierung in besonderen Situationen (Nachfragespitzen und Katastrophen). Damit entstehen Gestal- 
tungsfreiräume für die Spitäler. Die Analyse zeigt allerdings, dass die Spitäler diese Leistungsaufträge bislang nicht ausreichend spezifizieren und damit ein Instrument der Leistungsgestaltung und -steuerung verpassen.

Die spitalinterne Ausdifferenzierung der politischen Vorgabe $\mathrm{zu}$ einem quantitativen Leistungsauftrag sollte Breite, Tiefe, Qualitäten und Volumina der Notfalleistungen einschliesslich Katastrophenplanung sowie Lehre und Forschung in der Notfallmedizin berücksichtigen (vgl. Tab. 3). Dabei muss neben der politischen Vorgabe auch die Marktsituation (Konkurrenz, Stärken und Schwächen des eigenen Leistungsprofils) berücksichtigt werden.

- Die Breite des Leistungsauftrags definiert die Kompetenzfelder, welche eine Organisationseinheit abdecken muss. Für die Notfallstation wird mit der Auftragsbreite festgelegt, welche Patientinnen und Patienten aufgenommen werden.

- Mit der Tiefe des Leistungsauftrags wird festgelegt, welche Leistungen vor (z.B. Telefontriage) und nach den diagnostischen und therapeutischen Kernleistungen der Notfallmedizin angeboten werden sollen (z.B. Warte- und Entscheidungsräume).

- Als Qualitäten des Leistungsauftrags werden die medizinischen, serviceorientierten und wirtschaftlichen Sollwerte der Notfallmedizin festgelegt. Beispielsweise können maximale Wartezeiten, Fehlverlegungen, Reklamationsraten oder Umsatzwerte definiert werden.

- Mit den Volumina werden wirtschaftliche Zielgrössen vereinbart, welche den Ressourcenverbrauch rechtfertigen.

- Die Planung für besondere Ereignisse und Situationen wird zunehmend $\mathrm{zu}$ einem eigenständigen Auftrag für Notfallstationen: Bereitschaftskapazitäten, Mobilisierungsfristen und Katastrophenpläne sind zu definieren und sicherzustellen.

- Lehre und Forschung sind weitere Elemente des Leistungsauftrags, deren Quantifizierung für Ressourcenplanung und Controlling unverzichtbar ist.

Für alle diese Zielgrössen stehen Leistungsindikatoren zur Verfügung, die für Auftragsgestaltung und Controlling genutzt werden können (vgl. Tab. 2).

\section{Schlussfolgerungen und Empfehlungen}

Arbeitsteiligkeit erfordert zielgerichtete Koordination. Das trifft besonders für Notfallstationen $\mathrm{zu}$, deren Komplexität und damit Fehleranfäl- ligkeit von Technisierung, Variabilität und Vielfältigkeit der erforderlichen Leistungen bestimmt wird. Unter diesen Bedingungen kommt dem Leistungsauftrag als Basis für Organisation, Ressourcenzuordnung und Leistungssteuerung Bedeutung zu:

\section{Leistungsauftrag}

Konkrete Leistungsaufträge können die Planung der Notfallmedizin wesentlich unterstützen. Dabei sind Triage, Notfalldiagnostik, Notfalltherapie und Zuweisung zum nachfolgend behandelnden Team (Arzt, Abteilung, Institution) unbestritten. Weitere Aufgaben wie Vortriage (z.B. Telefontriage), Beobachtung (mehrere Stunden bis Tage) und der Betrieb von Warteund Entscheidungsräumen können der Notfallmedizin optional zugeordnet werden. Zunehmend werden auch Planung und Vorsorge für Nachfrageschwankungen und besondere Situationen (Katastrophen) dem Pflichtenheft der Notfallmedizin zugerechnet [26]. Angesichts dieses Aufgabenspektrums sind qualitative und quantitative Leistungsaufträge unverzichtbar.

\section{Kompetenzen}

Medizinische und logistische Aufgaben grenzen das Kerngebiet der Notfallmedizin von anderen medizinischen Spezialgebieten ab. Notfallmedizin erfordert spezielle und spezifische Fachkompetenzen, Teamfähigkeit sowie Leistungsfähigkeit unter Bedingungen limitierter Planbarkeit und dem Druck der Notfallsituation. Für diese Aufgaben müssen Notfallmedizinerinnen und -mediziner ausgewählt und strukturiert weitergebildet werden. Der Zugang zur Notfallmedizin muss von verschiedenen Facharzttiteln her möglich bleiben. Allerdings sollte die Notfallkompetenz durch einen Ausweis bestätigt werden.

\section{Organisation}

Die Notfallstation muss während 24 Stunden an 365 Tagen alle Notfallpatienten aufnehmen und nach Erstbehandlung den heute über 60 ambulanten und stationären Spezialisten zur Weiterbehandlung übergeben. Diese Koordinations- und Informationsaufgabe ist medizinisch und logistisch anspruchsvoll. Zudem stellen die multiprofessionellen Teams einer Notfallstation (Pflege, Ärzteschaft, Management) mit zahlreichen Schnittstellen und Schichtdiensten hohe Anforderungen an Planung, Führung, Weiterund Fortbildung. Diese Komplexität fordert organisatorische Eigenständigkeit und Entscheidungsautonomie. Die Einrichtung einer Organisations- 
einheit Notfallstation, die anderen Klinken und Abteilungen gleichgestellt ist, erscheint deshalb vorteilhaft.

\section{Leitlinien}

Notfallstationen müssen die Kompetenzen aller im Spital verfügbaren medizinischen Spezialisten nutzen, indem diese Fachleute die für das jeweilige Spital gültigen medizinischen Leitlinien festlegen. Die Leitlinienkompetenz muss bei den Experten der medizinischen Spezialgebiete liegen. Beispielsweise muss das Vorgehen bei einem gefässchirurgischen Patienten einschliesslich des Zeitpunkts und der Voraussetzungen für das Rufen des Gefässchirurgen von der Gefässchirurgie festgelegt werden. Im Sinne der Service-level-Vereinbarung muss vereinbart werden, unter welchen Voraussetzungen (Verdachtsdiagnose, Voruntersuchungen usw.) und innerhalb welcher zeitlichen Grenzen (Response-Zeit) ein klinischer Spezialist zur Verfügung steht.

\section{Kapazität}

Ausgelastete Notfallstationen sind überlastete Notfallstationen [27]. Notfallstationen müssen auch personelle und infrastrukturelle Kapazitäten für Vorhersage, Planung und Beherrschung besonderer Situationen (Grippewellen, Katastrophen) bereithalten [10]. Ausserdem müssen Notfallstationen über die Entscheidungskompetenz für den Einsatz dieser Ressourcen in einer ersten Katastrophenphase verfügen.

Der konkrete Leistungsauftrag einer Notfallstation muss die Kompetenzen und die Infrastruktur des Spitals ebenso wie dessen Umfeld (Einzugsgebiet, Konkurrenzsituation) berücksichtigen. Deshalb kann es keinen Standardauftrag geben. Der Leistungsauftrag sollte alle Qualitätsdimensionen adressieren und quantitative Ziele definieren, weil nur auf diesem Wege die Nachprüfbarkeit (Monitoring) gewährleistet werden kann. Der quantitative Leistungsauftrag ist als Beitrag zur Qualitätssicherung der Notfallversorgung einzustufen.

\section{Literatur}

1 Institute of Medicine. IOM report: The future of emergency care in the United States health system. Acad Emerg Med. 2006;13(10):1081-5.

2 Olshaker JS, Rathlev NK. Emergency Department overcrowding and ambulance diversion: The impact and potential solutions of extended boarding of admitted patients in the Emergency Department. J Emerg Med. 2006;30(3):351-6.
3 Fatovich DM, Nagree Y, Sprivulis P. Access block causes emergency department overcrowding and ambulance diversion in Perth, Western Australia. Emerg Med J. 2005;22:351-4.

4 Schull MJ, Vermeulen M, Slaughter G, Morrison M, Daly P. Emergency department crowding and thrombolysis delays in acute myocardial infarction. Ann Emerg Med. 2004;44:577-85

5 Miro O, Sanchez M, Espinosa G, Coll-Vinent B, Bragulat E, Milla A. Analysis of patient flow in the emergency department and the effect of an extensive reorganization. Emerg Med J. 2003;20: 143-8.

6 Weibel L, Gabrion I, Aussedat M, Kreutz G. Work-related stress in an emergency medical dispatch center. Ann Emerg Med. 2003;41(4) 500-6.

7 Gafner B, Zimmermann H, Hersperger M. Der ärztliche ambulante Notfalldienst im Kanton Bern 2006. Schweiz Ärztezeitung. 2007;88(5):174-9.

8 Division of Advocacy and Health Policy. A growing crisis in patient access to emergency surgical care. Bull Am Coll Surg. 2006;91:8-19.

9 McCarthy ML, Aronsky D, Kelen GD. The measurement of daily surge and its relevance to disaster preparedness. Acad Emerg Med. 2006;13:1138-41.

10 Jones SA, Joy MP, Pearson J. Forecasting demand of emergency care. Health Care Manag Sci. 2002;5: 297-305.

11 Stäubli M, Briner V, Hangartner PJ. Modell mit Nachteilen für Patientenbetreuung und Weiterbildung der Assistenzärzte. Schweiz Ärztezeitung 2006;87(35):1502-4.

12 Meier K, Arbeitsgruppe der Interessengemeinschaft ärztliche Leiter Notfallstationen. Notfallstationen: strukturelle und organisatorische Empfehlungen für die Qualitätssicherung. Schweiz Ärztezeitung. 2005;86(32):1918-28.

13 Plattform Rettungswesen FMH. Kategorien von Notfallstationen. Schweiz Ärztezeitung. 2005; 86(32):1915-7.

14 Mayring P. Qualitative Inhaltsanalyse. Weinheim, Basel: Beltz Verlag; 2003.

15 Cardin S, Afilalo M, Lang E, Collet JP, Colacone A, Tselios C, et al. Intervention to decrease emergency department crowding: Does it have an effect on return visits and hospital readmissions? Ann Emerg Med. 2003;41(2):173-85.

16 Hoffenberg S, Hill MB, Houry D. Does sharing process differences reduce patient length of stay in the emergency department? Ann Emerg Med. 2001;38(5):533-40.

17 Cronin JG, Wright J. Rapid assessment and initial patient treatment team - a way forward for emergency care. Accident Emerg Nurs. 2005;13:87-92.

18 Solberg LI, Asplin BR, Weinick RM, Magid DJ. Emergency department crowding: Consensus development of potential measures. Ann Emerg Med. 2003;42:824-34.

19 Panik A, Bokovoy J, Karoly E, Badillo K, Buckenmyer C, Vose C, et al. Research on the frontlines of healthcare. Nurs Res. 2006;55(2):S3-S9. 
20 Williams KA, Rose WD, Simon R. Teamwork in emergency medical services. Air Med J. 2007; 18(4):149-53.

21 Handler JA, Gillam M, Kirsch TD, Feied CF Metrics in the science of surge. Acad Emerg Med. 2006;13(11):1173-8

22 Asplin BR, Flottemesch TJ, Gordon BD. Developing models for patient flow and daily surge capacity research. Acad Emerg Med. 2006;13(11):1109-13.

23 Kellermann AL. Crisis in the emergency department. N Engl J Med. 2006;355(13):1300-3.

24 Welch S, Augustine J, Camargo CA, Reese C. Emergency department performance measures and benchmarking summit. Acad Emerg Med. 2006;13:1074-80.

25 Beattie E, Mackway-Jones K. A Delphi study to identify performance indicators for emergency medicine. Emerg Med J. 2004;21:47-50.

26 Werth G. Emergency departments overwhelmed, underfunded, and dangerously fragmented. Bull Am Coll Surg. 2007;92(3):20-6.

27 Bagust A, Place M, Posnett JW. Dynamics of bed use in accommodating emergency admissions: Stochastic simulation model. Br Med J. 1999;319: 155-8.
28 Schwappach DL, Blaudszun A, Conen D, Ebner H, Eichler K, Hochreutener MA. "Emerge": benchmarking of clinical performance and patients' experiences with emergency care in Switzerland. Int J Qual Health Care. 2003;15(6):473-85.

29 Richards DA, Meakins J, Tawfik J, Godfrey L, Dutton E, Heywood P. Quality monitoring of nurse telephone triage: pilot study. J Adv Nurs. 2004; 47(5):551-60.

30 DeVita MA, Bellomo R, Hillman K, Kellum J, Rotondi A, Teres D, et al. Findings of the first consensus conference on medical emergency teams. Crit Care Med. 2006;34(9):2463-78.

31 Taylor C, Benger JR. Patient satisfaction in emergency medicine. Emerg Med J. 2004;21:528-32.

32 Phillips S. Current status of surge research. Acad Emerg Med. 2006;13(11):1103-8.

33 Lynch T, Cox P. Reverse quality management: Developing evidence-based best practices in health emergency management. Qual Manag Health Care. 2006;15(2):104-15 